James Carole Lesley, Mackenzie Lynette Ann, 'Health professional's perceptions and practices in relation to functional capacity evaluations: Results of a quantitative survey', J ournal of Occupational Rehabilitation, 19 203-211 (2009) 


\section{TITLE PAGE.}

Title:

Health professional's perceptions and practices in relation to Functional Capacity Evaluations - results of a quantitative survey.

\section{Authors:}

Carole James $^{1}$ and Dr Lynette Mackenzie ${ }^{2}$

\section{Running Head:}

Health professional's perceptions \& practices to FCE: survey results.

\section{Correspondence:}

Carole James,

School of Health Sciences,

University of Newcastle,

University Drive,

Callaghan, NSW 2308 ,

Australia.

Tel: +61249216632

Fax: +61249216984

Email: Carole.James@newcastle.edu.au

1 School of Health Sciences, University of Newcastle, NSW, Australia.Email: Carole.James@newcastle.edu.au

2 School of Health Sciences, University of Newcastle,NSW, Australia.Email: I.mackenzie@usyd.edu.au 


\section{Abstract.}

Introduction: This study investigated the perceptions and practices of Australian health professionals in relation to the use of Functional Capacity Evaluations (FCE's)

Methods: A quantitative cross- sectional study design was used to survey health professionals who conduct FCE's and who were working for one of 219

rehabilitation providers in NSW, Australia. Seventy seven returned surveys were eligible for inclusion.

Results: 11 different FCE's were being utilised with many health professionals using more than one FCE. The most commonly used FCE was non-standardised (56\%, $n=43)$ followed by $52 \%(n=40)$ using the Workhab, and $18 \%(n=14)$ using Valpar.

Both non-standardised and standardised assessments were being used by $90 \%(n=69)$ of respondents.

Health professionals reported using all or parts of the FCE, and indicated identical FCE's are not always conducted, with adaptation of the FCE, due to client injury $(82 \%, n=62)$ and job $(80 \%, n=43)$ occurring. $60 \%$ of respondents had no choice in the type of FCE they conducted, and of the $40 \%$ with a choice, this was not influenced by other stakeholders in the process.

Accreditation and training, characteristics of assessment tasks, standardisation, reliability, cost, length and flexibility were all identified as factors affecting the selection of an FCE.

Conclusions: This study demonstrated that health professionals in NSW Australia, are not routinely using standardised tools for FCE's. Health professional perceptions suggest accreditation, training and the characteristics of the FCE were important factors in FCE selection. In practice, participants tended to use parts of an FCE rather than the whole FCE. Adaptation of FCE's was common, due to client injury and specific job requirements.

Keywords: Functional Capacity Evaluation, Occupational Rehabilitation. 


\section{Introduction.}

Within the practice areas of workplace injury management, occupational rehabilitation and work injury prevention, functional capacity evaluations (FCEs) are used to define an individual's functional abilities or limitations in the context of safe, productive work tasks [1]. Functional capacity evaluations are commonly used with individuals who have suffered work related musculoskeletal injuries as part of the rehabilitation process to remain or return to work. FCE's are designed to measure function, and therefore they can be used for a range of injury and disease types, both work and non work related. FCE's are used for work fitness determinations and to facilitate return to work[2] and have been identified as providing complementary information for insurance physicians assessing physical work ability[3]. FCE's are also used within the medico-legal arena[4].

There are a variety of FCE's available commercially and many clinics have developed their own nonstandardised, and work specific FCE's. All FCE's attempt to measure functional performance objectively, to produce reliable and valid results, however, studies to establish if this is achieved are limited[1,5]. Research in these areas, specifically on the reliability and validity of some of the commercially available assessments, is now beginning to be published [6-14]. A Standardised FCE can be defined as one that is commercially available, has acceptable measurement/ psychometric properties and is conducted using standardised procedures and protocols [4, 15]. A non-standardised FCE can be defined as a self designed, internally adaptable assessment tool which may be specifically designed for a particular industry. Commercially available FCE's have the common goal of identifying functional abilities however different tools use slightly different models and approaches. The main models in use are biomechanical, physiological, psychophysical and kinesiophysical [16]. Many FCE's are based on the physical domains outlined in the Dictionary of Occupational titles[1] and comprise a range of tasks that include these domains. Some tools include job simulation assessment; some include assessment of effort, symptom exaggeration, fear avoidance and other psychological issues as part of the FCE to determine safe lifting or physical demand capabilities.

Strong et al (2004) identified limitations of current research and difficulties of extrapolating information from a single point in time[17]. In a Canadian study, FCE's were conducted with variations in guidelines or practice standards, with limited contextual information, 
and practices were influenced by referral source and market demands[18].

Each country has it's own process and procedures in relation to occupational rehabilitation (defined as: a managed process that involves appropriate, adequate and timely services based on assessed needs, aiming to maintain an injured worker in, or return them to, suitable employment[19]), and as such differences in what and how assessments are being used is to be expected. As occupational rehabilitation falls under the workers compensation umbrella in many jurisdictions, legislation may influence requirements of this process, which will therefore vary across states and countries. FCE's are however, used for common purposes across the globe and commercially available standardised assessments are used across many different countries[4, 20]. There have been differences reported in the results of standardised FCE's between countries perhaps being the result of differences in patients or context[4], however, the practice issues facing the health professional in relation to FCE's are similar[18, 21, 22].

Therapists have identified flexibility of the assessment tool as a consideration when using an FCE, and this has an impact upon standardisation of a tool and it's reliability and validity[23, 24]. Several authors [1, 25] discuss the issue of generic versus specialist (or job specific) FCE testing, and the ability to alter the test depending upon the needs of the client, work and situation. Factors that have been identified as having an impact upon this adaptation include the reason for referral; client's job requirements; client's injury type; the therapists' workplace procedures, policies and resources; and the therapist's skill and experience [17, $18,21,23]$.

When looking at what specific assessments are being used within the occupational rehabilitation arena, Jundt and King (1999) completed a study of work rehabilitation programs in the USA, and found $94 \%$ of respondents conducted job analysis/risk hazard analysis and $91 \%$ conducted functional capacity evaluations. Of the FCE's being used, $45 \%$ used the Isernhagen work system, $12 \%$ the Ergos and $12 \%$ the Key system[26]. Deen et al (2002) looked specifically at occupational therapists (OT's), working in occupational rehabilitation settings in Australia and found $96 \%$ conducted workplace assessments, $86 \%$ functional capacity evaluations and $75 \%$ job/risk assessments. Of those conducting FCE's, specific tools being used were, Workhab-36\%, Valpar $23 \%$ and West - 18\%[27]. Cotton et al (2006) found $75 \%$ of rehabilitation providers in NSW conducted an "own design" FCE rather than a commercially available 
assessment tool and of those using commercial tools, Workhab was the most commonly used (17\%), followed by Isernhagen (10\%)[24].

Despite previous studies investigating FCE's and what assessments are being used, there is less data available about how FCE's are applied in practice.

This research aimed to build on existing research to identify the perceptions and describe the practice of Australian health professionals in relation to the use of FCE's.

\section{Method.}

Following ethics approval, a cross sectional study design was used to survey health professionals, who conduct FCE's, and who were working for rehabilitation providers in NSW. Only those health professionals who conduct FCE's were included in the study.

\subsection{Survey}

Items within the survey were developed from a qualitative study[23] which investigated the perceptions and practices of health professionals in relation to FCE usage, and using literature to identify assessment tools currently in use. The survey was piloted with twelve health professionals. The pilot survey was modified to improve the internal reliability of the survey subscales [28], and sent to participants in the mail.

The survey included 60 questions within six sections: Demographic data of the participants; Type of FCE used; FCE choice; FCE usage; Perceived consequences of using an FCE and Perceptions of FCE's (see Appendix 1 ). The survey included questions seeking responses both of a categorical nature (related to the background of the health professional and the type of FCE used). It included items about standardised FCE's, defined as those that are commercially available, have acceptable measurement/ psychometric properties and are conducted using standardised procedures and protocols [4, 15]; and non standardised FCE's, defined as self designed, internally adaptable assessment tools. Ordinal data relating to choice, usage, perceived consequences and perceptions of FCE's was also collected. These questions used direct estimation using likert scales. Raters expressed an opinion based on agreement with a series of statements about the use of FCE's[29]. Completed surveys were returned to the researcher by mail. 


\subsection{Participants.}

Health professionals who use FCE's and were working for WorkCover (NSW) accredited rehabilitation providers were invited to participate. Under the WorkCover(NSW) guidelines, Occupational therapists and Physiotherapists are approved to conduct FCE's. Other health professionals such as Exercise Physiologists and Occupational Health Nurses need WorkCover(NSW) approval to conduct these assessments under this regulatory system. In NSW, Australia, this consisted of 219 WorkCover (NSW) accredited rehabilitation providers, who may employ several health professionals too conduct FCE's.

\subsection{Procedure.}

The survey was sent to WorkCover (NSW) accredited rehabilitation providers. Accredited rehabilitation providers are organisations accredited by Workcover (NSW) to offer specialist services to help injured workers to return to work. Rehabilitation providers employ different health professionals to assess the needs of the injured worker and the workplace requirements, to then develop a rehabilitation plan to assist the injured worker to return to work [30]. The Managers of each rehabilitation provider were asked to distribute information about participating in the study to any health professionals who conducted FCE's within their organisation. Completed surveys were returned anonymously to the researcher in the mail.

\section{4. Data analysis}

Survey responses as either numerical, scaled items, categorical or nominal data, was entered into STATA [v8.0][31]. Data was checked after entering and missing variables were checked for accuracy prior to analysis. Descriptive analysis including mean values, confidence intervals and estimates of proportions were calculated.

\section{Results.}

\subsection{Participants:}

Eligible surveys were received from 77 participants working for 65 different rehabilitation providers, this being a response rate of $30 \%$ from the 219 rehabilitation providers invited to participate. Of those who replied, $82 \%(n=63)$ were occupational therapists, $13 \%(n=10)$ physiotherapists and $5 \%(n=4)$ exercise physiologists. 
This response is generally representative of the ratio of the different professionals working for rehabilitation providers in NSW.

The mean number of years of professional experience was 10.9 years (range:-22yrs) and the mean number of years of FCE experience was 5.3 years (range:1-16yrs).

Table I provides a description of the sample and shows the number of professionals involved in the study, yrs of experience by profession and years of FCE experience by profession.

\section{Insert table I here.}

\subsection{Practice issues:}

\subsubsection{FCE's in use in NSW.}

The results indicated 10 standardised FCE's were being used throughout NSW. Additionally health professionals described using non-standardised FCE's in practice. Health professionals reported all the assessments they were currently using, and therefore could indicate more than one assessment. The most frequently used FCE was a non-standardised assessment $(56 \% n=43)$. Both nonstandardised and standardised FCE's were being used by $46 \%(n=35)$ of health professionals and $91 \%(n=69)$ were using standardised FCE's. Of the standardised assessments being used the most frequently used was the Workhab $(53 \%, n=40)$, followed by the Valpar assessment $(18 \%, n=14)$.

Non standardised assessments only, were used by $10.5 \%$ $(n=8)$ of health professionals. $44 \%(n=34)$ used only a standardised assessment, and the remaining health professionals reported using both a non-standardised and standardised assessment - 46\% $(n=35) .26 \%$ of all respondents $(n=20)$ were using more than one type of standardised assessment. Of these, $13 \%(n=10)$ were using two different standardised assessments, $12 \%(n=9)$ were using three different standardised assessments and one $(1.3 \%)$ health professional reported using five different standardised assessments.

Table II outlines FCE usage by profession.

Insert table II here.

The seven most commonly used FCE's in NSW were: Non-standardised assessments, Workhab, Valpar, Isernhagen, West, Pile and Keys in descending order of popularity.

\subsubsection{Patterns of FCE Utilisation.}


Health professionals identified that at times they used all of the components of the assessment and at times they only used parts of the assessment - for both nonstandardised and standardised assessments. The proportions of health professionals using part or all of the components of the seven most popular assessments is outlined in Figure $I$ \& 2. Health professionals could indicate pattern of use for more than one FCE.

\section{Insert Fig. 1 \& 2 here}

Forty percent $(40 \%, n=31)$ of health professionals indicated they were unlikely to use identical FCE's with all clients, whereas $36 \%(n=28)$ indicated they would. Of those respondents $(n=43)$ who used a non-standardised assessment $34 \%(n=15)$ indicated they were unlikely to use identical FCE's and the same amount $(34 \%, n=15)$ would. Of those health professionals $(n=69)$ using standardised assessments, $40 \%(n=28)$ indicated they were unlikely to use identical FCE's and $36 \%(n=25)$ indicated they would use identical assessments. Health professionals indicated they adapted the FCE conducted in response to the clients' injury $(82 \%, n=63)$, and $80 \%$ $(n=43)$ indicated they would adapt the FCE in response to the clients' job.

\subsubsection{Frequency of use.}

Figure 3 shows the frequency of use of the seven most popular standardised assessments identified. $56 \%$ $(n=43)$ of health professionals indicated that they used a non-standardised assessment. Of these $68 \%(n=28)$ used this less than twice per month and $31 \%(n=13)$ indicated they used this more than three times a month.

Insert Fig. 3 here.

\subsubsection{FCE's and Workplace assessments}

Out of the total participant group, 33\% $(n=25)$ of health professionals indicated for each client, that they frequently conducted an FCE (either standardised or non-standardised) along with a workplace assessment. A further $47 \%(n=36)$ of health professionals indicated they often conducted Workplace assessments instead of FCE's for individual clients.

\subsection{Perceptions in regard to FCE's.}

\subsubsection{Factors affecting selection of FCE.}

Regardless of preferences in use of FCE's in NSW, Australia, the importance of different criteria in the 
selection of an FCE identified accreditation \& training to conduct the assessment and the characteristics of the assessment tasks as highly important, and standardisation, reliability, cost, length and effort as moderately important. This is outlined in Table III.

Insert Table III here.

$40 \%(n=31)$ of Health Professionals indicated they had a choice in the type of FCE that was conducted. $60 \%$ $(n=46)$ had no choice, suggesting that only one assessment was available to them.

Of those health professionals with a choice of FCE type, $64 \%$ indicated they were the only person choosing the FCE. Choice of FCE used was not influenced by the: referrer, client, doctor, the health professionals' employer or the clients' employer for the majority of health professionals. Figure 4 shows the frequency of different influences on the choice of FCE used, for those health professionals that did have a choice.

Insert Fig. 4 here.

\section{Discussion.}

4.1. Practice issues.

4.1.1.FCE's in use in NSW.

Despite the wide range of FCE's available, most health professionals continue to use a non-standardised FCE, as identified in previous research [24] The use of nonstandardised assessments raises questions in regards to what and how aspects of function are being assessed and the consistency of these assessments. The expectation that health professionals are using evidence to inform practice, can therefore be questioned as it raises the issue that evidence relating to the properties of many FCE's, especially non-standardised tools, is lacking. Amongst those that used a standardised FCE, most used the Workhab (52\%) and, as was identified by Cotton et al, the two most commonly used are the least expensive to purchase[24]. Workhab was also identified as the most commonly used FCE in research by Deen [27]. Workhab is an Australian developed \& produced tool which may impact upon it's popularity. The other commercially available tools identified in this study have all been developed internationally, and are identified as less commonly used in Australian studies, including this one $[24,27]$.

Many health professionals had a choice in the FCE they used, with $44 \%$ indicating they used both a non- 
standardised and a standardised FCE, however when they chose to use one over the other was not identified. Use of more than one type of standardised tool was also found in $26 \%$ of health professionals however the reasons they chose a specific standardised assessment for any particular client was not clarified. Of the health professionals who had a choice in the FCE used, $64 \%$ indicated they were the only person choosing the FCE, suggesting health professionals with a choice had the most control over which FCE they used. Having the ability to choose a specific FCE also allows increased professional judgement on the part of the health professional. Clinical reasoning skills to determine the most appropriate FCE tool, based on the model of FCE, the client injury, client job and purpose of the assessment may impact upon this choice, however it may also be the result of personal preferences and perceptions relating to FCE's as discussed later.

Availability at the workplace was rated highly by health professionals in this study, which concurs with the study by strong et al [17]. Availability is also a factor in relation to choice of FCE type. In this study, $60 \%$ of health professionals did not have a choice in the type of FCE that was conducted, this links with previous research conducted in NSW Australia, where health professionals were found to use only one FCE tool, however it was not indicated in that research whether the health professionals had a choice and chose to only use one FCE or whether these health professionals did not have a choice[24].

Choice may be limited by the availability of FCE tools with a particular rehabilitation provider and economic factors may influence this, as a result of the cost of purchase of the assessment tool, and additional costs of training and accreditation for the health professional to use the tool.

\subsubsection{Patterns of FCE utilisation.}

Health professionals identified that they were using parts of, rather than the whole assessment in many instances. Depending upon the actual assessment tool, using part of the assessment can impact upon the standardisation of the tool and therefore may impact upon the reliability and validity of the tool.

Of the commercially available standardised FCE's the health professionals using the Keys assessment were the most likely to use all components of the tool. It is difficult to know with a non-standardised FCE what is the whole or a part of the assessment, however the 
responses from participants indicated that they used parts of the tool, suggesting the assessment comprises a variety of components.

Health professionals adapted FCE's to suit specific requirements, especially in relation to the clients' injury type or job. This concurs with other authors [1, 25] who identified the issue of generic versus specialist (or job specific) FCE testing, and the ability to alter the test depending upon the needs of the client, work and situation. The use of, or lack of use of identical FCE's was identified in this study, with adaptation of an assessment for a specific client commonly being reported. This has been identified in previous research $[1,25]$ and also relates to the issue of the clinical utility of the assessment tool [32].

For those health professionals who said they would conduct identical assessments, this could suggest a lack of alternatives or a more standardised approach. This has implications in relation to standardised protocols being used with FCE's regardless of client presentation. Similar percentages of users (using both nonstandardised and standardised FCE's) indicated they conducted identical FCE's, which does not suggest using a standardised FCE results in more standardised protocols and procedures being used, as might be expected.

\subsubsection{Frequency of Use and Workplace Assessments}

FCE's were reported to be used several times a month however, health professionals also reported conducting a workplace assessment in place of or in conjunction with an FCE. Under the Workcover (NSW) occupational rehabilitation guidelines a workplace assessment is required for those clients returning to the workplace[30] and it would therefore be expected most clients would undergo a workplace assessment, whereas a functional assessment is not mandatory and therefore only completed on some clients. This may be associated with the type of client involved in the rehabilitation process, and whether they have a job to return to or not. Previous research has suggested a workplace assessment is desirable to measure ability for a specific job [22], and identified three types of work related assessment workplace assessments that are specific to the work place and work tasks, functional capacity evaluations for those with a job and thirdly for those without a job[21]. Further research could investigate the relationship between those injured workers who undergo a FCE and a workplace assessment or only a work related assessment 
to determine if there is a link between an FCE and those clients who have a job to return to or not.

\subsection{Perceptions in relation to FCE's.}

Health Professionals in this study rated accreditation and training to conduct an FCE highly. This suggests that FCE's are perceived as a specialised area of practice that requires the health professional to undertake specific training. This is the case with some standardised FCE's that require health professionals to: undergo training; obtain accreditation; and in some instances have ongoing requirements to maintain accreditation. All health professionals responded that accreditation/ training was important; for those health professionals who use a non-standardised assessment tool, for which there is no accreditation, it can be presumed that training was considered important and is required. WorkCover (NSW) recognises minimum qualifications of professionals to conduct any FCE's (standardised or non standardised). For occupational therapists or physiotherapists, this is a minimum of 3 months occupational rehabilitation experience. Other health professionals are required to undergo an assessment process, to be eligible to conduct FCE's under the WorkCover (NSW) system [30].

Health professionals in this study did not identify reliability and validity of assessment tools as an area of concern nor did they identify standardisation as particularly important, which could be directly related to the fact that many health professionals were using nonstandardised FCE's. Research based appropriate assessment tools are generally considered essential to ensure credible practice, however as Clemson and Fitzgerald [33] found issues of reliability and validity were not clearly understood by therapists which may account for this not being rated highly in this study. It is interesting to note that specific FCE's are not being requested by the referring agencies. This suggests the issues of the measurement properties including the reliability and validity of an assessment tool is not an important consideration for these referrers nor for the health professionals in their own choice of an assessment tool.

It is important to note, that much FCE research, particularly about the reliability and validity of specific assessment tools, is only just beginning to become available for some of the commercially available assessment tools. Therefore health professionals need to be reviewing and updating their knowledge to assist in providing the best possible, evidence based care for 
clients. With a large percentage of health professionals using non-standardised assessments, the issues

relating to the measurement properties of the tools are of more concern. Further research related to specific FCE's is needed, to provide evidence of the measurement properties and reliability and validity in relation to their usage. This is particularly important in an age when health professionals are being encouraged to justify treatments and services with evidence, both by the health professions as a whole and by those paying for services.[34]

Task characteristics and flexibility within the FCE, were rated as important criteria by health professionals, in the choice of the FCE and these relate to the adaptation of the FCE. Flexibility within the assessment can threaten the standardised application of the assessment tool, which may also account for standardisation not being rated highly in this study.

Cost of the assessment, length and effort to conduct the FCE were all rated as moderately important in this research. Within the Workcover (NSW) occupational rehabilitation system, [17] FCE's are charged to insurers and employers based on the time taken to complete, and costs are discussed and approved by the referrer prior to the service being provided in this environment. Effort required to conduct the assessment was rated least important when looking at the different qualities of the FCE, suggesting the health professional is motivated to put in more effort if needs are being met. These needs could be related to those of the client and his or her rehabilitation, or related to the needs of the health professional in fulfilling their work requirements and managing their case load.

The issues of time, effort, length, flexibility and task characteristic will also inform the health professional inregard to the choice of FCE used (where choice is available). Further research could investigate the reasons health professionals choose one FCE over another and investigate the issue of effort, motivation and the health professional in relation to the occupational rehabilitation process.

\section{Conclusions}

This study identified that despite different FCE assessment tools being used in NSW Australia, a nonstandardised tool was preferred by most participants. 
Many health professionals adapted FCE's by only using parts of them, or changing items in response to the client injury and job. Standardisation, reliability and validity were identified as important criteria when participants selected FCE's. However, task

characteristics and flexibility within the FCE were also identified as important criteria; leading to increased adaptation of FCE's to suit individual clients, thus impacting on the standardisation, reliability and validity of the FCE's.

Despite the limitations of this study being a small sample and the sample being from one state within Australia, the results provide a profile of usual practice in relation to FCE usage within this environment.

Further research to investigate what components of an FCE are being included or not, or under what circumstances this occurs in practice is recommended.

\section{Acknowledgements}

The authors would like to thank all health professionals who gave their time to participate in this study.

\section{References.}

[1] King PM, Tuckwell N, Barrett TE. A critical review of functional capacity evaluations. Physical Therapy. $1998 ; 78(8): 852$.

[2] Gross D, Battie M, Asante A. Evaluation of a Short form Functional Capacity Evaluation: Less maybe Best. Journal of Occupational Rehabilitation. 2007;17:422-35. [3] Wind H, Gouttegbarge V, Kuijer P, Sluiter J, FringsDresen M. Complementary value of functional capacity evaluation for physicians in assessing the physical work ability of workers with musculoskeletal disorders. International archives of Occupational Environmental Health. 2008(Oct 9).

[4] Reneman M, Kool J, Oesch P, Geertzen J, Battie M, Gross D. Manual handling performance of patients with chronic low back pain during Functional Capacity Evaluation: A comparison between three countries. Disability and Rehabilitation. 2006;28(18):1143-9. [5] Innes E. Reliability and Validity of Functional Capacity Evaluations: An Update. International Journal of Disability Management Research. 2006;1(1):135-48. [6] Durand $M-J$, Brassard B, Hong Q, Lemaire J, Loisel $P$. Responsiveness of the Physical Work Performance Evaluation, a Functional Capacity Evaluation, in Patients 
with Low Back Pain. Journal of Occupational Rehabilitation. 2008;18(1):58-67.

[7] Boadella J, Sluiter j, Frings-Dresen MHW.

Reliability of Upper Extremity Tests Measured by the Ergos Work Simulator: A Pilot Study. Journal of

Occupational Rehabilitation. 2003 December;13(4):21932 .

[8] Durand M-J, Loisel P, Poitras S, Mercier R, Stock $S$, Lemaire J. The interrater reliability of a Functional Capacity Evaluation: The Physical Work Performance Evaluation. Journal of Occupational Rehabilitation. 2004 June;14(2):119-29.

[9] Jay M, Lamb J, Watson R, Young I, Fearon F, Alday $J$, et al. Sensitivity and specificity of the indicators of sincere effort of the EPIC lift capacity test on a previously injured population. Spine. 2000 Jun $1 ; 25(11): 1405-12$.

[10] Matheson L, Rogers L, Kaskutas V, Dakos M. Reliability and reactivity of three new functional assessment measures. Work. 2002;18:41-50.

[11] Reneman M, Brouwer S, Meinema A, Dijkstra P, Geertzen J, Groothoff J. Test-retest reliability of the Isernhagen Work Systems Functional Capacity Evaluation in healthy adults. Journal of Occupational Rehabilitation. 2004 Dec2004///;14(4):295-305. [12] Ting W, Wessel J, Brintnell S, Maikala R, Bhambhani Y. Validity of the Baltimore Therapeutic Equipment Work Simulator in the measurement of lifting endurance in healthy men. American Journal of Occupational Therapy. 2001 Mar-Apr;55(2):184-90. [13] Reesink D, Jorritsma W, Reneman M. Basis for a Functional Capacity Evaluation Methodology for Patients with Work-related Neck Disorders. Journal of Occupational Rehabilitation. 2007;17(3):436-49.

[14] Gouttebarge V, Wind H, Kuijer P, Frings-Dresen $M$. Reliability and Validity of Functional Capacity Evalutation methods: a systematic review with reference to Blankenship, Ergos work simulator, Ergo-Kit and Isernhagen work system. International Archives of Occupational Environmental Health. 2004 November $2004 ; 77: 527-37$.

[15] Innes E, Straker L. Workplace assessments and functional capacity evaluations: Current beliefs of therapists in Australia. Work. 2003;20:225-36.

[16] Gibson L, Strong J. Expert review of an approach to functional capacity evaluation. Work. 2002;19:231-42.

[17] Strong S, Baptiste S, Cole D, Clarke J, Costa M, Shannon $H$, et al. Functional assessment of injured workers: A profile of assessor practices. The Canadian Journal of Occupational Therapy. 2004 Feb;71(1):(13$23)$. 
[18] Strong S, Baptiste S, Clarke J, Costa M. Use of functional capacity evaluations in workplaces and the compensation system: A report on workers' and users' perceptions. Work. 2004;23:67-77.

[19] WorkCover(Victoria). Occupational Rehabilitation Service Standards. WorkCover Victoria 2006.

[20] WorkHab I. About WorkHab FCE Systems. [Website] 2008 [cited 2009020209 ]; Available from:

http://www.workhab.com/

[21] Innes E, Straker L. Workplace assessments and functional capacity evaluations: Current practices of therapists in Australia. Work. 2002;18(1):51-66.

[22] Pransky G, Dempsey P. Practical Aspects of

Functional Capactiy Evaluations. Journal of

Occupational Rehabilitation. 2004 Sept;14(3):217-29.

[23] James C, MacKenzie L, Higginbotham N. Health

Professionals' Attitudes and Practices in relation to

Functional Capacity Evaluations. Work. 2007;29(2):81-8.

[24] Cotton A, Schonstein E, Adams R. Use of

Functional Capacity Evaluations by rehabilitation

providers in NSW. Work. 2006;26:287-95.

[25] Innes E, Straker L. Attributes of excellence in work related assessments. Work. 2003;20:63-76.

[26] Jundt J, King P. Work rehabilitation programs: a

1997 survey. Work. 1999;12:139-44.

[27] Deen M, Gibson L, Strong J. A survey of

occupational therapy in Australian work practice. Work.

$2002 ; 19: 219-30$.

[28] Higginbotham N, Albrecht G, Connor L. Health

Social Science: A transdisciplinary and complexity

perspective. Melbourne: Oxford Press 2001.

[29] Portney LG, Watkins MP. Foundations of Clinical

Research: Applications to practice. 2nd ed. New Jersey:

Prentice Hall Health 2000.

[30] WorkCover(NSW). Rehabilitation Providers.

Standards and conditions of Accreditation. In: NSW W, ed.: Workcover NSW 2002.

[31] Statacorp. StataStatistical Software: Release 8.0.

8.0 ed. College Station, TX: Stata Corporation 2003.

[32] Toomey M, Nicolson D, Carswell A. The clinical utility of the Canadian Occupational Performance

Measure. Canadian Journal of Occupational Therapy.

1995 December 1995;62(5):242-9.

[33] Clemson L, Fitzgerald M. Understanding

assessment concepts within the occupational therapy

context. Occupational Therapy International.

$1998 ; 5(1): 18-34$.

[34] Muir Gray J. Evidence-based Healthcare: How to make Health Policy and Management Decisions. 2nd ed. London: Churchill Livingstone 1997. 
Table I - Descriptive information on sample $(N=77)$

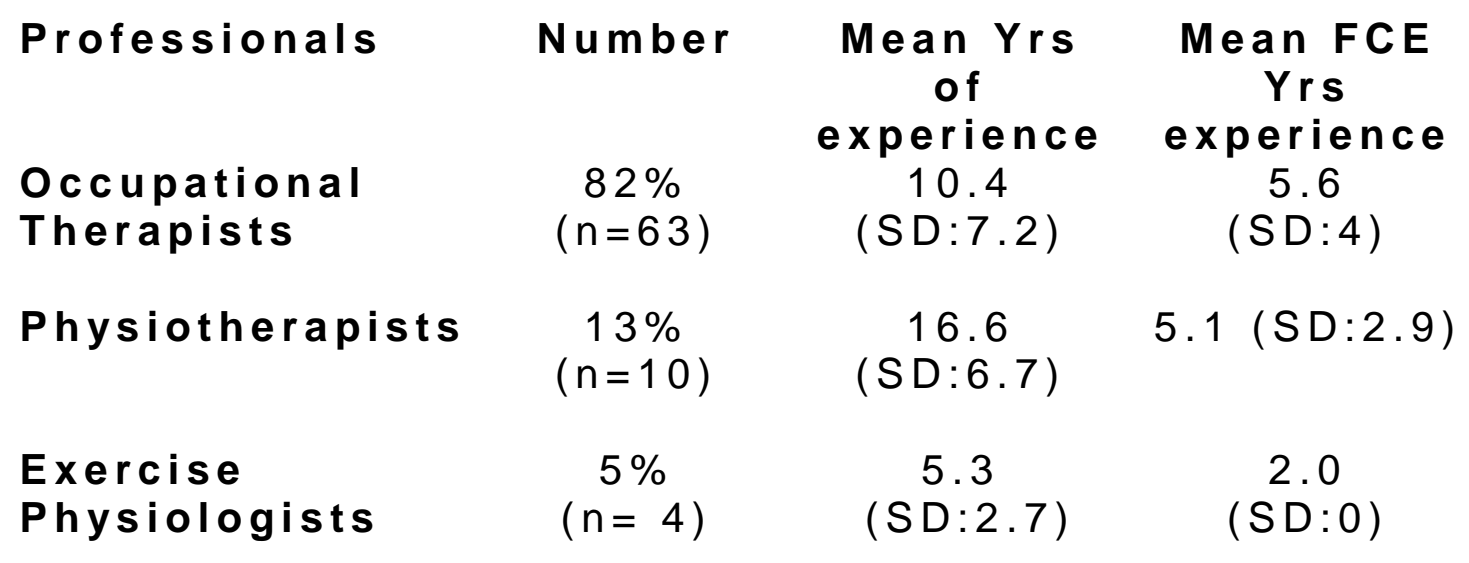


Table II: Usage by FCE type and Profession $(N=77)$.

\begin{tabular}{|c|c|c|c|c|}
\hline FCE & $\begin{array}{l}\text { No's } \\
\text { OT's } \\
(N=63)\end{array}$ & $\begin{array}{c}\text { No's } \\
P T^{\prime} s \\
(N=10)\end{array}$ & $\begin{array}{l}\text { No's } \\
\text { ExPh } \\
(N=4)\end{array}$ & $\begin{array}{c}\text { Total } \\
\text { Number } \\
(N=77)\end{array}$ \\
\hline $\begin{array}{l}\text { Non } \\
\text { Standardised }\end{array}$ & $33(52 \%)$ & $6(60 \%)$ & $4(100 \%)$ & $43(56 \%)$ \\
\hline $\begin{array}{l}\text { Non } \\
\text { Standardised } \\
\text { + Standardised }\end{array}$ & $29(46 \%)$ & $4(40 \%)$ & $2(50 \%)$ & $35(46 \%)$ \\
\hline Standardised & $59(94 \%)$ & $8(80 \%)$ & $2(50 \%)$ & $69(91 \%)$ \\
\hline Standardised F & CE by type: & & & \\
\hline Workhab & $35(56 \%)$ & $4(40 \%)$ & $1(25 \%)$ & $40(53 \%)$ \\
\hline Valpar & $14(22.5 \%)$ & 0 & 0 & $14(18 \%)$ \\
\hline Isernhagen & $10(16 \%)$ & $3(30 \%)$ & 0 & $13(17 \%)$ \\
\hline Pile & $8(12.9 \%)$ & 0 & $1(25 \%)$ & $9(12 \%)$ \\
\hline West & $9(14.5 \%)$ & 0 & 0 & $9(12 \%)$ \\
\hline Keys & $6(9.7 \%)$ & $1(10 \%)$ & 0 & $7(9 \%)$ \\
\hline Blankenship & $2(3.2 \%)$ & $2(20 \%)$ & 0 & $4(5 \%)$ \\
\hline PWPE & $4(6.5 \%)$ & 0 & 0 & $4(5 \%)$ \\
\hline Ergos & 0 & $1(10 \%)$ & 0 & $1(1.3 \%)$ \\
\hline Workability & $1(1.6 \%)$ & 0 & 0 & $1(1.3 \%)$ \\
\hline
\end{tabular}

* Health professionals could select more than one. 
Table III: Mean score of importance of different qualities/ criteria in choice of FCE $(N=77)$

\begin{tabular}{|lc|}
\hline Criterial Quality & $\begin{array}{c}\text { Mean Score } \\
(\mathbf{0}-\mathbf{5})\end{array}$ \\
\hline Availability & $3.9(\mathrm{SD}: 1.3)$ \\
Flexibility & $3.8(\mathrm{SD}: 0.8)$ \\
Standardisation & $3.6(\mathrm{SD}: 1.1)$ \\
Reliability/Validity & $3.6(\mathrm{SD}: 1.0)$ \\
Task Characteristics & $4.0(\mathrm{SD}: 0.7)$ \\
Accreditation/training & $4.2(\mathrm{SD}: 0.8)$ \\
Length & $3.4(\mathrm{SD}: 0.9)$ \\
Cost & $3.5(\mathrm{SD}: 0.9)$ \\
Effort & $3.0(\mathrm{SD}: 1.1)$ \\
\hline
\end{tabular}


Fig 1: Utilisation of ALL components of standardised FCE $\left(n=71^{*}\right)$.

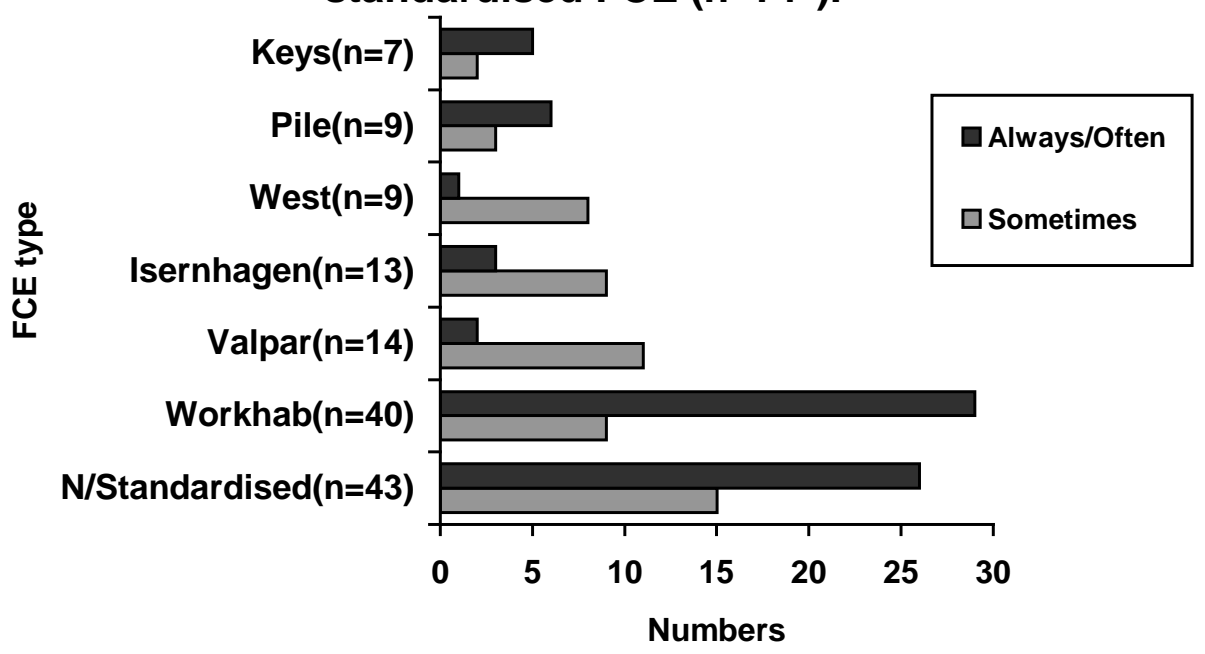

- Health professionals could report on more than one FCE.

- *6 missing variables. 
Fig 2: Utilisation of PARTS of FCE $(n=60)$ Numbers

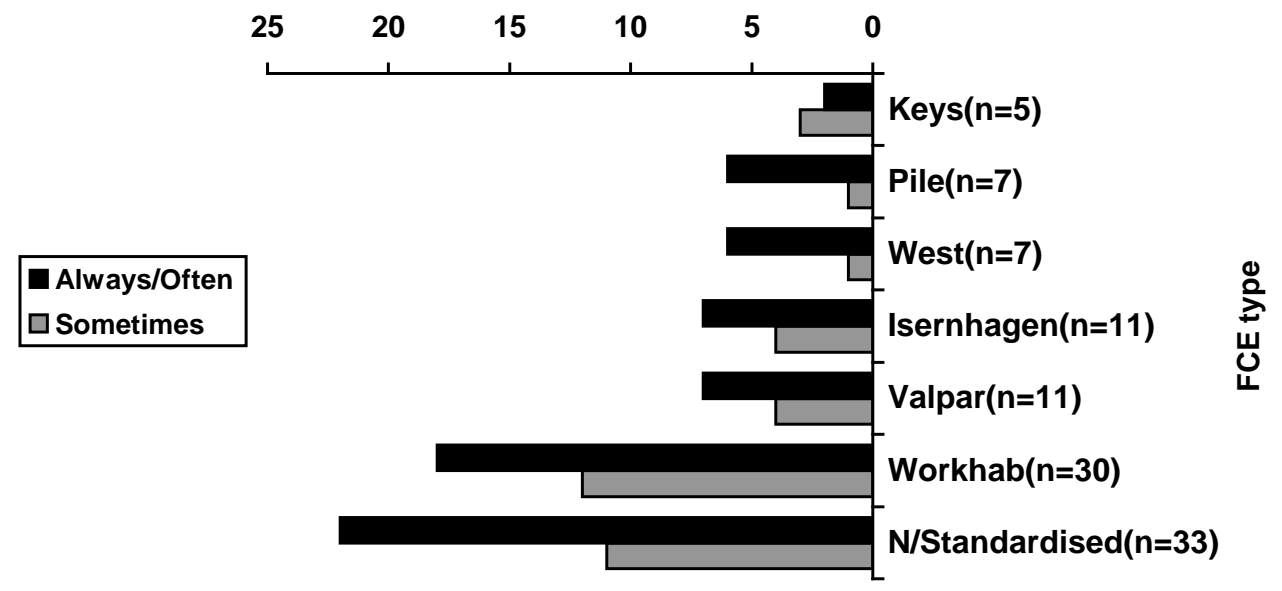

- Health professionals could report on more than one FCE. 
Fig 3: Frequency of use (\%/ month) of most popular Standardised FCE's $\left(n=76^{*}\right)$

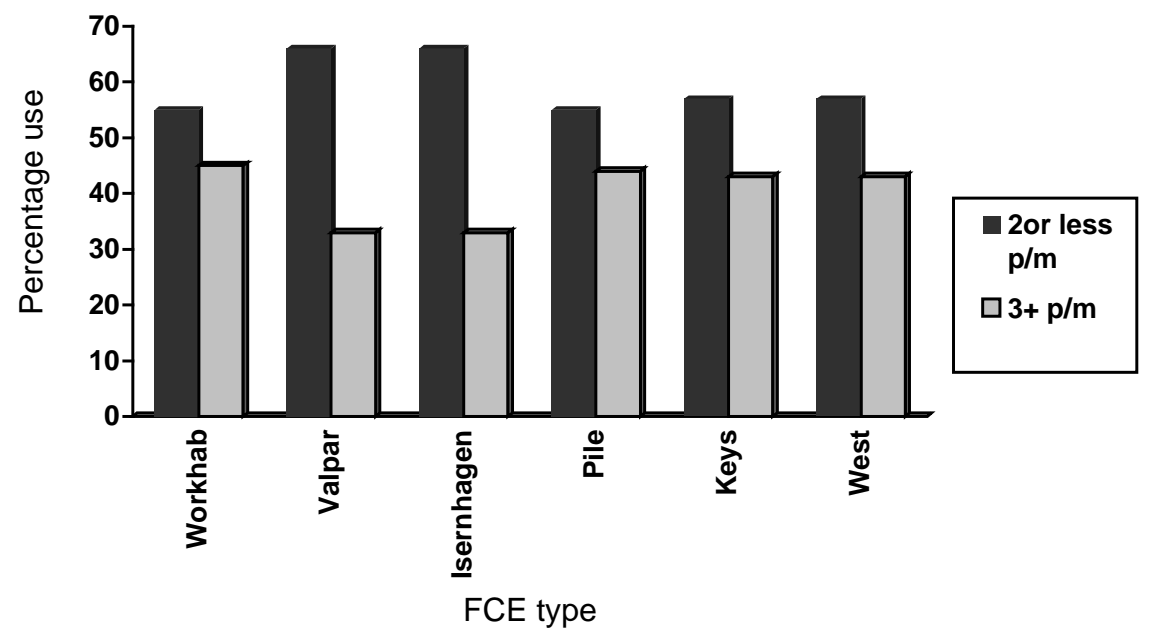

- Health professionals could report on more than one FCE.

- * 1 missing variable. 
Fig 4: Influences for choice of FCE's ( $n=31)$

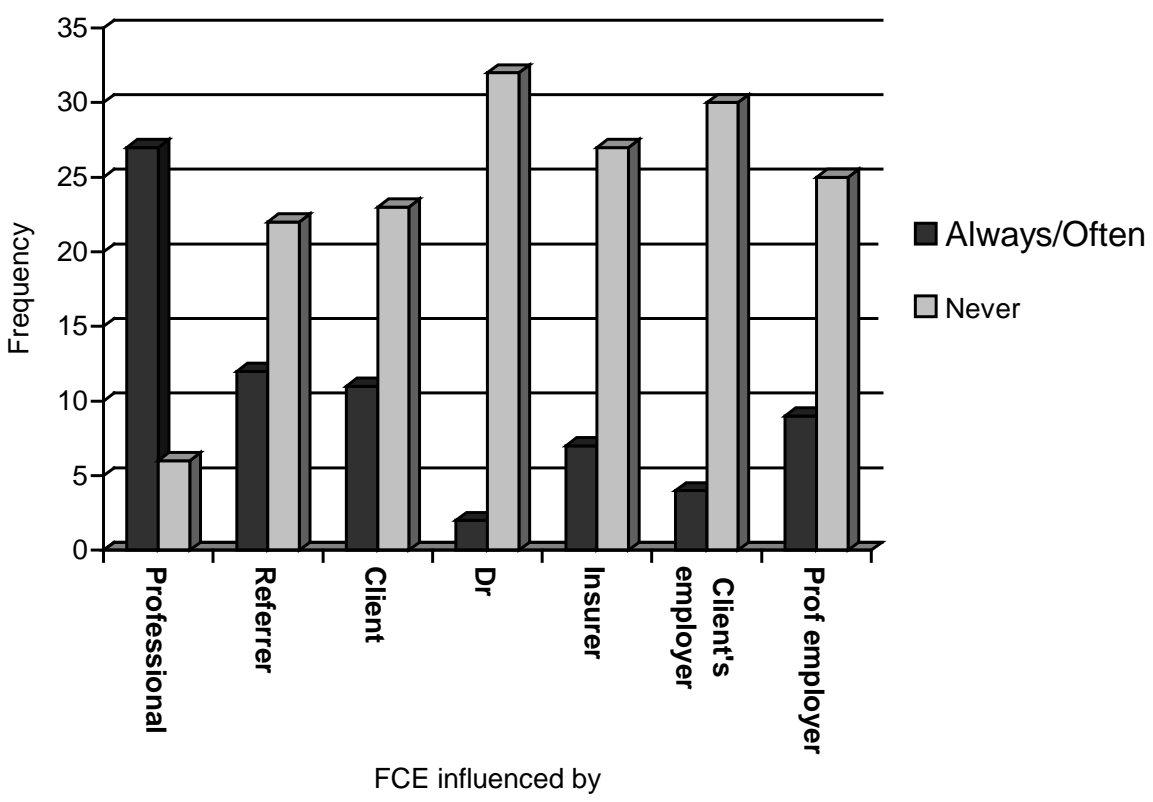

